Bull. Mater. Sci., Vol. 5, Nos $3 \&$ 4, August \& October 1983, pp. 219-230.

(C) Printed in India.

\title{
The kinetics of pressure-induced polymorphic transformations
}

\begin{abstract}
A K SINGH
Materials Science Division, National Aeronatical Laboratory, Bangalore 560 017, India

Abstract: The various methods of obtaining the kinetics data in pressure-induced transformations have been briefly reviewed. Some of the recent results obtained for the pressureinduced alpha-omega transformation in titanium, and foc-boc tränsformation in ytterbium have been discussed. The general features of the kinetics data have been compared with those for other transformations available in literature.
\end{abstract}

Keywords. High pressure; kinetics; phase transformation; titanium; ytterbium

\section{Introduction}

The study of the kinetics of phase transformation often plays an important role in understanding the mechanism of the phase transformation. The kinetics of the pressure-induced transformations have not been studied as extensively as the kinetics of temperature-induced transformations mainly because of the difficulties in high pressure experiments. The transformations studied in the past were mostly of reconstructive type (a classification introduced by Buerger 1953) which require high temperature and high pressure. Some of the transformations studied in detail are: calcite $\rightleftharpoons$ aragonite (Davis and Adams 1965; Brar and Schloessin 1979); $\mathrm{GeO}_{2}(\alpha$. quartz) $\rightleftharpoons \mathrm{GeO}_{2}$ (rutile) (Zeto and Roy 1969; Brar and Schloessin 1981); $\mathrm{PbO}_{2}(\mathrm{I}) \rightleftharpoons \mathrm{PbO}_{2}$ (II) (Dachille and Roy 1964); $\gamma \rightarrow \alpha$ transformation in $\mathrm{Fe}_{2} \mathrm{O}_{3}$ (Goto $1964)$; graphite $\rightleftharpoons$ diamond (Hanneman 1969; Bundy 1969). The earlier studies on the kinetics of pressure-induced phase transformations have been reviewed by Onodera (1971), and Osugi et al (1975). In this paper the discussion is restricted to the polymorphic transformations which occur at room temperature. The results obtained under isobaric conditions for alpha-omega transformation in titanium and $\mathrm{foc}-\mathrm{bcc}$ transformation in ytterbium have been discussed.

\section{General considerations}

The relative stabilities of the various phases (of the same chemical composition) can be conveniently discussed with reference to a diagram showing the variation of Gibbs free energy, $G$, as a function of the thermodynamic variable. Such a diagram for a system thich exhibits two phases $A$ and $B$ as the pressure is varied, is shown in figure 1 . The lines marked 1 and 2 depict the variation of $G$ with pressure for respectively phases $A$ Ind $B$. The point of intersection of the two lines represents the pressure, $p_{0}$, at which the free energies of the two phases are equal; $p_{0}$ is termed thermodynamic equilibrium Tressure. The phase $A$ is stable below $p_{0}$ whereas phase $B$ is stable above $p_{0}$. The Trmbols $A_{m}$ and $B_{m}$ indicate metastable phases.

In practice the transformation $A \rightarrow B$ does not take place at $p_{0}$, but of ten a pressure 


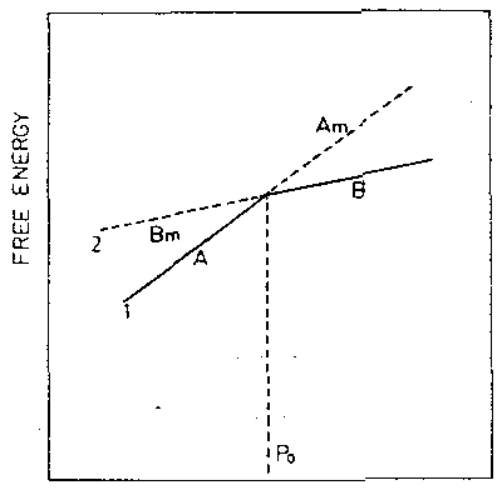

PRESSURE

Figure 1. Variation of Gibbs free energy with pressure of phases $A \operatorname{aod} B$ in the vicinity of $p_{0}$. Phaw A undergoes a first order transformation to phase $B$ above $p_{0}$.

well above $p_{0}$ has to be applied to drive the transformation. This is because the transformation faces an activation barrier (figure 2). The activation free energy $\Delta G^{*}$ is often very large compared to the free energy difference bet ween thetwophases $A$ and $B$, Phase A which may be present above $p_{0}$ is, in fact, a metastable phase $A_{m}$. Similarly, when the pressure is reduced from well beyond $p_{0}$ where only phase $B$ is present, phase A does not reappear until the pressure is reduced well below $p_{0}$. Phase $B$ which persists below $p_{0}$ is also ametastable state. The differencebetween $p_{0}$ and the pressure requited to observe $A, \rightarrow B$ transformation $\uparrow$ is called over-pressure, and depends on the magnitude of $\Delta G^{*}$. The larger the $\mathrm{AG}^{*}$ the higher will be the over-pressure required for a given rate of transformation.

The presence of an activation barrier (and hence the requirement of an over-pressure to drive the transformation) implies that any physical property measured with increasing and decreasing pressure around $p_{0}$ will exhibit a hysteresis. This hysteresis is inherent to the transformation and is different from the hysteresis arising from the friction in high pressure set-up. A hypothetical plot of a physical property against pressure exhibiting a hysteresis is shown in figure 3 . The different phases and the pressure range over which they exist are also indicated. It may be noted that the magnitude of the property of phase $\boldsymbol{A}$ measured with decreasing pressure does not always coincide with that obtained with increasing pressure. The properties such as electrical conductivity which may depend on the grain size and the plastic strains in the specimen show a value, on cycling through the transformation, which differ from the Starting value. However, some other properties, such as volume change under pressure, show reproducible values with increasing or decreasing pressures in the region where either phase A or $B$ alone is present.

In conmoniy used notations this transformation is simply denoted by $A \rightarrow B$, but strictly speaking it is $A_{m} \rightarrow B$. The $A \rightarrow A_{m}$ involves only an increase in $G$ along the line 1 in figure 1 , and does not represent a phase transformation. The suffix $m$ is therefore just a reminder that phase $A$ is already in the region where $B$ is stable. 


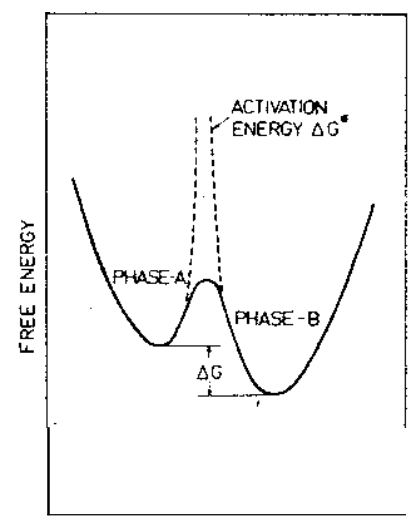

TRANSFORMATION PATH

Figure 2. A section at $p>p_{0}$ indicating the variation of free energy along the transformation path.

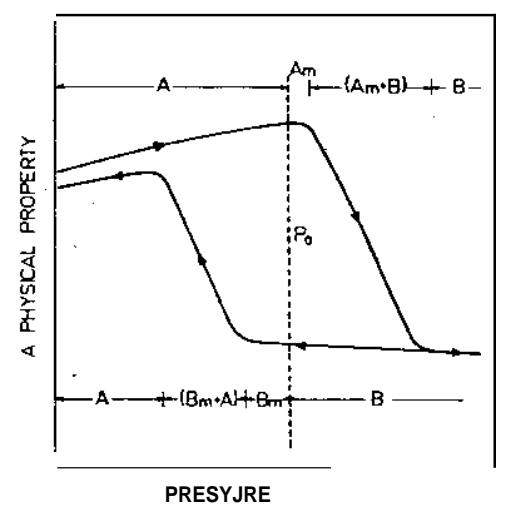

Figure 3. Intrinsic hysteresis when a physical property is measured with increasing and decreasing pressure enclosing $A \rightleftharpoons B$ phase transformation.

Seen from figure 3 that $p_{0}$ lies within the hysteresis loop. The position of $p_{0}$ with ot lo the hysteresis loop is important because this knowledge can be used to imentally determine $p_{0}$ which is an important parameter. It is often assumed that ocated at the midpoint of the hysteresis loop. Thus, $F_{0}$ in these experiments have laken as the average of the pressures at which $A_{m} \rightarrow B$ and $B_{m} \rightarrow A$ are observed to However, there is no a priori justification for this. As discussed earlier, the overure required to produce an observable rate of transformation depends on the tion free energy for the transformation. Thus, $p_{0}$ can lie at the midpoint of the 
hysterisis loop only if the activation barriers for the $A_{m} \rightarrow B$ and $B_{m} \rightarrow A$ transformations are identical. In general, the position of $p_{0}$ remains to be determined by more elaborate experiments.

The method of determining $p_{0}$ was suggested by Bridgman (1940). The pressure is increased in small steps and the progress of the transformation is monitored by observing the change in specimen resistivity for a fixed interval of time after each increase. If $\boldsymbol{A}, \rightarrow \boldsymbol{B}$ transformation is observed, then the pressure is decreased in steps with a waiting period after each decrement of pressure, until $\boldsymbol{B}_{\boldsymbol{m}} \rightarrow \boldsymbol{A}$ transformation is observed. The process is repeated until a region is obtained within which no perceptible $A_{m} \rightarrow$ B or $B_{m} \rightarrow$ A transformation takes place. This region was termed "region of indifference- by Bridgman. It is then assumed that $p_{0}$ lies in the middle of the region of indifference. This method requires that the pressure be truly hydrostatic and be measured in situ by suitable method, for example with the help of a manganin gauge. This method is timesonsuming and has been used only for a few transformations which are used as pressure standards (Zeto et of 1968; Zeto and Vanfleet 1969). This method poses serious experimental problems at pressures above $6 \mathrm{GPa}$. As a result it is customary in high pressure work to quote the pressure at which the transformation is detected with increasing pressure. The transformation pressure thus obtained is termed 'start.pressure'. The start pressure is not related to $p_{0}$ in any definite manner. The next best that has been done is to assume that $p_{0}$ is located at the midpoint of the hysteresis.

The determination of $p_{0}$ by the above method breaks down in case of transformations which exhibit such large hysteresis that the high pressure phase is metastably retained at atmospheric pressure. In such cases, the width of the hysteresis can be reduced by intentionally increasing the shear component of stress while pressurizing the specimen. The determination of $p_{0}$ of $\alpha \rightarrow \omega$ transformation in titanium is one such example (Zil'Bershteyn et al 1976). As the temperature ofthe specimen is increased the hysteresis in $a \rightarrow \omega$ transformation decreases Taking advantage of this fact Zil'Bershteyn et al (1974) determine $p_{0}$ at higher temperatures by takingtheaverageof the $\alpha \rightarrow \omega$ and $\omega \rightarrow$ a transformation pressures. The value of $p_{0}$ at room temperature was obtained from these measurements by extrapolation of the $p_{0}$ versus temperature data. The $p_{0}$ for $\alpha \rightarrow \omega$ transformation in titanium obtained by the two methods agrees well. Both the methods, however, assume that $\boldsymbol{p}_{\mathbf{0}}$ is located at the midpoint of the hysteresis loop.

The high pressure transformations may occur from one stable to another stable phase, stable to metastable phase, or even irom one metastable phase to another metastable phase. A number of interesting examples are given by Roy (1969). In this paper the discussion will be confined to transformations from one stable phase to another stable phase; this may, however, involve a metastable phase as an intermediate step.

The pressure-induced transformations which have been studied for their kinetics aspects fall under twocategories. In the first category are polymorphic transformations which are displacive transformations (Buerger 1953). These transformations usually have low activation energy and are relatively rapid. Sincepolymorphic transformations can coout at relatively lower temperatures (room temperatures or lower), any diffusion process is unlikely to take place. The second category of transformations are the reconstructive type. These have large activation energy and are generally sluggish. The transformation rates become appreciable only at higher temperatures. Such transformations are often diffusion-controlled 


\section{Experimental methods}

The study of the kinetics of phase transformations requires information on the fraction transformed as a function of time. The change in a physical property such as volume or the electrical resistance of the specimen is recorded as a function of time as the transformation progresses. The fraction transformed at various time intervals are calculated from such data. It is desirable to obtain the kinetics data under isobaric conditions because the interpretation of the data is simpler. However, it is not uncommon to find in literature studies made under the conditions of varying pressures. The methods commonly used to obtain the kinetics data are described in some detail.

The measurement of volume change associated with a first order transformation offers a simple method of obtaining the kinetics data. The fraction transformed is proportional to the volume decrease; the constant of proportionality is obtained by noting the volume decrease for complete transformation. A piston-cylinder high pressure set-up is ideally suited for such measurements. The sensitivity of the measurements is considerably improved in these experiments because a rektively largesized specimen (volume $\% \mathrm{l} \mathrm{cm}^{3}$ ) can be used. As the transformation progresses and the volume decreases, the piston moves inward. For a properly designed set-up the volume change is proportional to thepiston displacement Thus, complete kinetics data can be obtained hy recording the piston displacement as a function of time. This method however, has a serious drawback; the large size of the specimen, which resultsin an increased sensitivity in the measurement of volume changes, leads to a large drop in pressure when transformation takes place. It is difficult to maintain a constant pressure during the transformation. For this reason, studies under isobaric conditions cannot be made. In spite of this drawback, the method has been used to obtain interesting results (Livshi $z$ et al 1960, 1969; Lacam et al 1973; Leliwa Kopystynski et al 1975).

The change in electrical resistance of the specimen can be used to monitor the progress of the transformation. A typical resistance-pressuredata for titanium is shown in figure 4. As the pressure is increased the resistance of $\alpha$-titanium decreases. and with

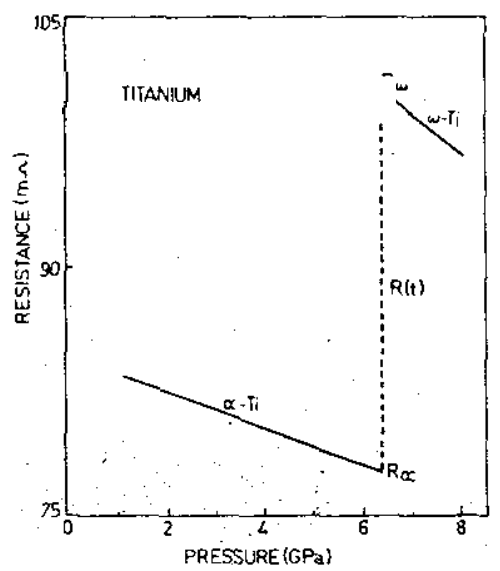

Figure 4. The resistance of titanium specimen as a function of pressure 
sufficientlyhigh over-pressure the transformation to $\omega$-phase starts. The resistance of the specimen $\mathrm{R}(t)$ becomes time-dependent, and is recorded as a function of time. The resistances of the specimen in $\alpha$ - and o-phase at a constant pressure are respectively $R_{\alpha}$ and $\mathrm{R}$, In general, $\alpha$ and $\omega$-phase may be taken to denote respectively the low and high pressure phase in a pressure-induced transformation. The fraction of o-phase formed at any instant $\mathrm{t}$ can be calculated from the knowledge of $\mathrm{R}(t), R_{\dot{\alpha}}$ and $\mathrm{R}$, In fact $\mathrm{R}(t)$ represents the resistance of an intimate mixture of $\boldsymbol{a}$ and $\omega$ phases. If it is assumed that resistances of the $\boldsymbol{a}$ and $\omega$ phases lie in series, then it turns out that the fraction of $w$ phase is proportional to the change in resistance. This approximation has often been used to calculate the fraction transformed. Clearly, this assumption represents an extreme case, and is not likely to have a general validity. It can as well be assumed that the $\alpha$ and o-phases provide a parallel resistive path, and in such a case the fraction transformed is proportional to the change in electrical conductivity. A rigorous expression for the effectiveresistance of a two-phase random mixture is given by Landauer (1952). This expression, instead ofa series or parallel approximation, has been used in recent studies to estimate the fraction transformed (Singh et al 1982a, 1982b). More complicated models have been used in other studies (Lombos and Mahdaly 1977).

The resistometric method ofdetermining the fraction transformed has an advantage that a small specimen $(05 \times 005 \times 5 \mathrm{~mm})$ is used. The volume change oftransformation is a very small fraction of the volume of the high pressure cell, and therefore the pressure does not drop as the transformation progresses. This makes it possible to obtain data under isobaric conditions.

The transformations resulting in quenchable phases can be studied by $\mathrm{x}$-ray diffraction technique. This involves holding the specimens at a given temperature and pressure for different times and quenching them to room temperature and one atmosphere. The fraction of the high pressure phase is determined at one atmosphere by $\mathrm{x}$-ray diffraction technique. The high intensity $\mathrm{x}$-ray sources, such as rotating anode $\mathrm{x}$-ray generators and synchrotron radiation source, make it possible to record diffraction patterns from specimen under pressure in a few minutes. Thus, it is possible now to determine the fraction transformed by pressurizing the specimen and carrying out in situ X-ray diffraction analysis. This method has been recently used to study the $B 1 \rightarrow B 2$ transformation in $\mathrm{KCl}$ (Hamaya and Akimoto 1981). The precision with which the fractions. of the phases can he determined is rather limited.

It is instructive to compare the various methods discussed above and point out their merits and drawbacks. The method based on the measurement of volume change directly gives the fraction transformed, hut it is difficult to maintain the pressure constant. Further, since a piston-cylinder apparatus is used, the highest pressure is limited to a b u t $4.5 \mathrm{GPa}$. The resistometric methods are most convenient in practice. Since the specimen size is small, the progressoftransfonnation does not result inadrop in pressure. Since the specimen size is small, it is possible to use opposed anvil devicesto pressurize the specimen. These devices using tungsten carbide anvils can easily go up to $15 \mathrm{GPa}$ This method however can be used only with conducting or semiconducting specimen. The calculation of the fraction transformed assumes that specimen is an intimate random mixture of two phases. Any departure from this in actual case is likely to introduce егrors. The x-ray methods are more cumbersome in general, and less precise when used with high pressure x-ray cameras.

The study of kinetics under isobaric conditions requires that the specimen be 
pressurized to a desired level before any transformation can take place. Far sluggish transformations the loading rates available in conventional high pressure set-up are adequate. However, for fast transformations a specially designed loading set-up is required. A method for rapidly loading an opposed anvil set-up was developed, and is showr schematically in figure 5 . The bomb, the hydraulic ram and the reservoircan be Fisolated by closing respectively $V_{1}, V_{2}$ and $V_{3}$. The pump has a built in non-return valve. The hydraulic ram which loads a tungsten carbide opposed anvil set-up is first pressurized to a desired initial pressure by closing $V_{3}$ and opening $V_{1}$ and $V_{2}$. The ram is isolated by closing $V_{2}$. The bomb is pressurized to a higher pressure. The pressure in the hydraulic ram jumps to a tigher value when $V_{2}$ is suddenly opened. The final ram pressure is a function of initial pressure and the bomb pressure. The rise time of the pressure pulse is nearly $200 \mathrm{msec}$. Such a rapid pressurization results in a temperature rise of the specimen hut it is small and can be neglected. The details of this method are given elsewhere (Singh et al 1983a).

The change in other physical properties associated with the transfonnation, has been occasionally used to follow the progress of the transformation. Samaraet al (1967) studjed the rate of orthorhombic to cubic transformation in thallium iodide by monitoring the change in the specimen capacitance. The changes in optical absorption of transparent specimen can be used to study the kinetics of transformations. Takano et al (1982) used the diamond anvil cell to this effect.

\section{Results and discussion}

The literature on the kinetics of pressure-induced transformations is rather limited.The first measurements of the rates of solid-solid transformations were made by Bridgman (1916) by following the rate of decrease in pressure caused by the decrease in volume associated with the transformation. It was observed that the rate of transformation increased with increasing over-pressure.More recently, while characterizing accurately $\mathrm{BiI} \rightarrow$ II transition for the purpose of pressure calibration, Davidson and Lee (1964), and Zeto et al (1968) studied the kinetics aspect. It becane clear that the transformation was not a fixed-strain transformation, but involved a thermally activated nucleation process. Further, the transformation was isobaric in that it ran to completion as the

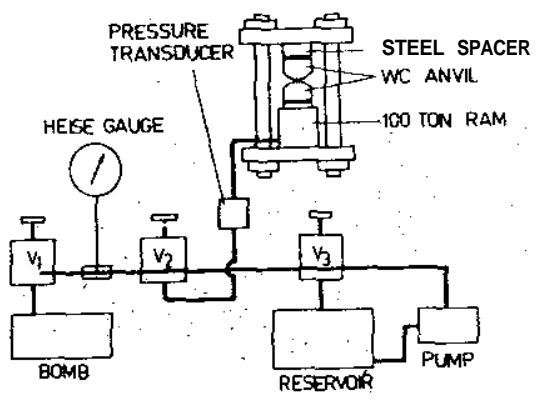

Figre 5. A method for step-loading an opposed anvil set-up. 
pressure was held constant. Though these studies were significant in establishing the nature of the transformation, no quantitative kinetics studies were made. Kopystynski et al (1975) studied the B1-B2 transformation in rubidium chloride by measuring the pressure drop in the high pressure cell $\boldsymbol{\infty}$ the transformation progressed. The rate of pressure drop could be explained on the basis of a nucleation and growth model. Hamaya and Akimoto (1981) studied the B1-B2 transformation in potassium chloride by pressurizing the specimen in a cubic press and using insit $u \mathrm{X}$-ray diffraction analysis. They showed clearly that the kinetics data satisfied Avrami equation. Then appeared the results of the studies on kinetics of $\alpha \rightarrow$ otransformationin titanjum (Singh et al 1982b; Murali Mohan et al 1982; Singh et al 1983a), and foc-be transformation in ytterbium (Singh et of $1982 \mathrm{~b}$ ). These studies reveal some interesting features of the displacive transformations, and will be discussed further. The isobaric conditions were achieved either by a rapid pressurization or by a special loading technique (Singh et al 1983a). The progress of the transformation was followed by monitoring the resistance of the specimen. The fraction of the high pressure phase as a function of time was calculated from the specimen-resistance versus time data. The following features were observed in both titanium and ytterbium transformations.

(i) The transformations started with a definite incubation (induction) period. The incubation period decreased rapidly with increasing over-pressure A similarbehaviour wasobserved by Onodera (1971) in cadmium chalcogenides. Onodera (1971) suggested that the observed incubation period could be the intrinsic property of the transition or could arise as a result of time delay in pressure transmission by solid pressure transmitting medium. The results on titanium and ytterbium transformations indicate that the observed incubation period is the inherent property of the transformation.

(ii) The transformations are isobaric-isothermal type in that these run to completionas the pressureis beld constant. This is inagreement with the earlier observation (Vohra et al 1977)that the transformation progressed with time as the pressure was held constant. Similar behaviour was observed in bismuth (Davidson and Lee 1964) and potassium chloride (Hamaya and Akimoto 1981). In cadmium chalwgenides the transformation was found to start under isobaric conditions initially (Onodera 1971) but tailed off after some time; either a change in temperature or pressure was required to drive the transformation to completion.

(iii) The kinetics data satisfied Avrami equation (Christian 1975);

$$
\zeta=1-\exp -\{t / \tau(p)\}^{n(p)},
$$

where $\zeta$ is the fraction of the high pressure phase at an instant $t$, and $\mathrm{T}$ and $n$ are constants at a given pressure, but in general are functions of pressure. At $\mathrm{t}=\tau, \zeta=1$ $-\exp (-1) \approx 063$. Thus, $\tau$ is the time required for nearly $63 \%$ transformation. This equation is derived assuming that the transformation involves the steps of nucleation and growth. In the case of homogeneous nucleation which decreases with time, $3<n$ $<4,2<n<3$ and $1<n<2$ for, respectively, a three-, two-, and onedimensional growth. The case $n=1$ represents a homogeneous rate equation. In polymorphic transformations. the lowest value of $n$ that can arise is unity, and occurs for growth of nuclei formed on grain boundaries after site saturation (Christian 1975).

In the case of $\alpha-\omega$ transformation in titanium $n=3 \pm I$ at $5 \mathrm{GPa}$ and decreases to $n=1 \pm 0.2$ at $9 \mathrm{Gpa}$. For foc-bce transformations in ytterbium, $n=1 \cdot 1 \pm 0.2$ at 3.4 $\mathrm{GPa}$ and decreases to $n=0-3 \pm 0-1$ at $4.8 \mathrm{GPa}$. As mentioned above, the lowest value of $n$ predicted fron theoretical models in polymorphic transformation is unity. 
However, experimentally, $n<1$ has been observed. Obviously, an improved model of nucleation is required to deal with this situation.

The parameter $\tau$ in (I) is found to decrease rapidly with increase in pressure. The pressure dependence of $\tau$ is shown in figure 6 . The In $\tau$ versus pressure data is linear far ytterbium, but shows a small curvature in titanium. In terms of the activation free energy for the combined process of nucleation and growth, 7 is given by

$$
\ln \tau(p)=b_{0}+\Delta G^{*}(p) / R T,
$$

where $b_{0}$ is aconstant, $\mathrm{AG}^{*}(\rho)$ is the pressure-dependent activation freeenergy, and $\mathrm{R}$ is gas constant. Equation (2) suggests that $\Delta G^{*}(p)$ can be obtained from the measurement of $\tau(p)$ if $b_{0}$ is known. The calculation of $b_{0}$ from first principles is complicated. The following arguments can be used toestimate the value of $b_{0}$. For a given value of $b_{0}$, th $\tau$ decreases with decreasing $\Delta G^{*}(p)$, and assumes a minimum value when $\Delta G^{*}(p)=0$. It may be noted that negative value of $\Delta G^{*}(p)$ has no physical significancein the present context. In the absence of any energy barrier, the transformation will propagate with velocity of sound. The velocity of sound in solids is typicallya few $\mathrm{km} / \mathrm{sec}$. This suggests that $\tau \approx 10^{-6}$ sec when $\Delta G^{*}(p)=0$, and $b_{0}=-13 \cdot 8$. This is the largest value of $b_{0}$ for which $\tau=10^{-6}$ sec can occur. For $b_{0}$ less than $-13 \cdot 8, \tau=10^{-6}$ sec can occur for a positive value of $\Delta G^{*}(p)$

It is seen from (2)that the pressure dependence of $\Delta G^{*}(p)$ is essentially the pressure. dependence of $I_{n} \tau$, for a constant $b_{0}$. The variations of $\Delta G^{*}$ with pressure as obtained from (2) with $b_{0}=-13.8$ are shown in figure 7 for titanium and ytterbium. The activation free energy is nearly $15 \mathrm{kal} / \mathrm{mol}$ at $5 \mathrm{GPa}$ for $\mathbf{a}-0$ transformation in titanium, and at $4 \mathrm{GPa}$ for foc-bce transformation in ytterbium. In bothcasesactivation free energy decreases with increasing pressure.

The expesimentally obtained values of activation free energies are significantly lower than the activation energy for self diffusion. This suggests that diffusion process is not mvolved in these transformations. In diffusioncontrolled transformations, the activation free energy is expected to increase or temain relatively unaltered when pressure is increased. The fact that activation free energy decreases with increase in pressure in the present case, also suggests-the absence of any diffusion process in the transformation.

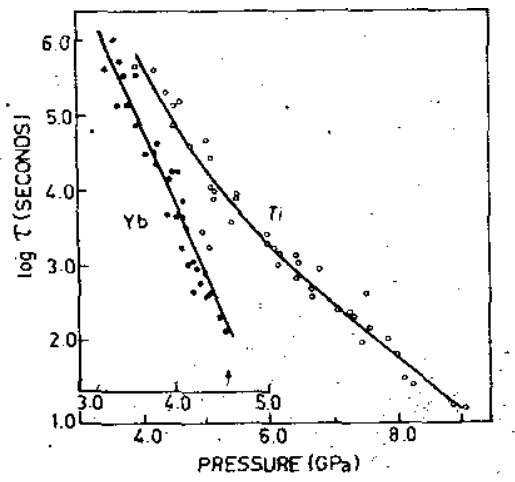

Figure 6. The pressure dependence of $\tau$ for titanium and ytterbium 


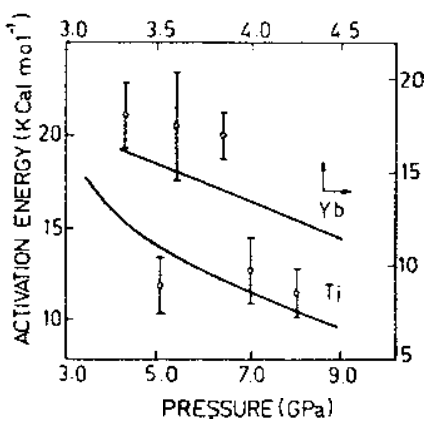

Figure 7. The activation free energy for the total process as a function of pressure. The dots indicate measured activation enthalpies.

Similar conclusions have been drawn in case of transformation in potassium chloride (Hamaya and Akimoto 1981) and cadmium chalcogenides (Onodera 1971).

The experiments were also carried out at elevated temperatures and $i$-values determined. The activation enthalpies were determined from $\ln \tau$ versus $\$ / T$ plot. The values ofactivation enthalpies thus obtained together with the error bars are marked in figure I. From the differences between the activation free energies and the corresponding enthalpies, the activation entropies wereestimated, and found to be $4 \mathrm{cal} / \mathrm{mol} / \mathrm{K}$-for titanium and $8 \mathrm{cal} / \mathrm{mol} / \mathrm{K}$ for ytterbium.

It is seen from figure 6 that the transformations in both casesoccur over a wide range of pressures. In principle transformation can occur at any pressure above-equilibrium pressure. The present data indicate that $p_{0}<4.0 \mathrm{GPa}$ for $\alpha \rightarrow \omega$ transformation in titanium, since experimentally the transformation has been found to occur even at $4 \mathrm{GPa}$. This is consistent with the experimental value of $p_{0}=2 \mathrm{GPa}$, as obtained by Zil'Bershteyn et al (1976). For the Same reasons, the present data suggest that $p_{0}<3.4 \mathrm{GPa}$ for ytterbium. No measurement of $p_{0}$ for fec-boc transformation in ytterbium seerns to have been made.

Inview ofthexkineticsdataonemayask thequestion: what is the significance of the experimentally determined transformation pressure or often termed start pressure? It appears that start pressure is a function, among many other parameters such as specimen history and the impurity levels, of the rate of loading, and is pressureat which incubation period becomes of the order of the time of loading.

\section{Transformation under sbock loading}

The oocurrence of $a \rightarrow \omega$ transformation in titanium under shock loading was clearly established by Kutsar et al (1974). The x-ray examination of the specimens, shockloaded to $35 \mathrm{GPa}$ at $293 \mathrm{~K}$, indicated the presence ofo-phase in traces. The estimated shock residual temperature at such high pressures exceeded the temperature for $\omega \rightarrow \boldsymbol{\alpha}$ transformation, which resulted in a poor yield of the $\omega$-phase. On cooling the specimen down to $120 \mathrm{~K}$ before loading it to $35 \mathrm{GPa}$, nearly $60 \% \omega$-phase was detected.

The transformation under shock loading can occur only if its kinetics becomes fast 
enough, under the pressure-temperature condition of shock loading, for the system to respond to the loads of short duration, typically a few $\mu$ sec. Extrapolation of $t-p$ data suggests that $\tau$ becomes of the order ofpsec at $20 \mathrm{GPa}$. Thus, the extrapolation of the present data suggests that nearly $63 \%$ o-phase can be obtained by shock loading the specimens to $20 \mathrm{GPa}$. This is about half the pressure at which Kutsar et al (1974) observed the transformation under shock loading. However, it is not clear from the work of Kutsar et al (1974) whether the experiments were systematically conducted at lowe shock pressures and the occurrence of o-phase ruled out. McQueen et al (1970) reported a discontinuity in the shock Hugoniot at $17.5 \mathrm{GPa}$ with an associated volume change of about $1 \%$ The specimens recovered after shock loading were stated to be in bcc phase, It may be noted that the first five lines of the o-phase can be indexed on the basis of boc lattice. If the shock-recovered specimens were in the $\omega$-phase, then the discontinuity in the shock Hugoniot can beattributed to $\alpha \rightarrow \omega$ transformation, and the pressure of transformation $(17.5 \mathrm{GPa})$ would agree very well with the present extrapolated value of $20 \mathrm{GPa}$.

The discussion so far was confined to the estimation of lowest shock pressures which will lead to $63 \%$ o-phase. $\boldsymbol{A}$ smaller fraction of $\%$-phase can be expected at lower pressures. The lowest shock pressure required to produce a given fraction of o-phase was estimated using In $t_{\zeta}-p$ data $\left(t_{\zeta}\right.$ is the time for a fraction oftransformation $\zeta$ ) to determine the pressure at which $t_{\zeta}$ becomes $1 \mu$ sec. These estimates suggest that detectable fraction of $\omega$-phase, say $10 \%$, can occur at shock pressures as low as $16 \mathrm{GPa}$.

In these discussions the effect on the $\alpha \rightarrow \omega$ transformation of the temperature which occursduring the shock loading (McQueen and Marsh 1960) has been neglected If the residual temperature, i.e. the specimen temperature after the shock is reduced to zero, exceeds the $\omega \rightarrow$ a transformation temperature $(410-430 \mathrm{~K})$ then a reverse transformation may occur resulting in a low fraction ofo-phase in the specimens recovered after shock compression. The estimated residual temperature (MoQueen and Marsh 1960) after shock loading to $20 \mathrm{GPa}$ is about $350 \mathrm{~K}$, which is less than the $\omega \rightarrow \bar{x}$ transformation temperature. For this reason it appears reasonable to neglect the temperature effect in the present discussions. However, even a $20-30 \mathrm{~K}$ rise in temperature is found to speed up the kinetics considerably. Thus a temperature rise in front of the shock wave is likely to enhance the kineties of $x \rightarrow Q$ transformation and thereby faci'itating the formation of o-phase.

The extrapolation of the in $t$ versus pressure data for ytterbium suggests that $\tau=10^{-6} \mathrm{sec}$ at $7 \mathrm{GPa}$, indicating that the foc bec transformation is likely to oceur at $7 \mathrm{GPa}$ under shock loading. However, there. is no shock wave data for ytterbium available for comparison.

\section{Acknowledgements}

The author wishes to thank $\boldsymbol{C}$ 'Divakar and Murali Mohan for many helpful discussions.

\section{References}

Btar N S and Schioessin H H 1979 Can J. Earth Sci 161402

Brir N S and Schloessin H H 1981 High Temp. High Pressures 13313 
Brjdgman P W 1916 Proc. Am. Acad. Arts. Sci. 5257

Bridgman P W 1940 Proc. Am. Acad. Anr. Sci. 741

Buerger M J 1953 Phase transformations in solids (New York John Wiley' pp 183

Bundy F P 1969 Reactivity of solids (ed.) J W Mitchell et al (New York Wiley-Interscience) pp 817

Christian J W 1975The theory of transformations in metals and alloys Part I Equilibrium and general kine

theory (New York Pergamon)

Dachille F and Roy R 1964J. Geol. 72243

Daridson T E and Lee A P 1964 Trans. Met. Soc AIME 2301035

Davis B L and Adams L H 1965 J. Geophys. Res. 10433

Goto Y 1964 Jpn J, Appl, Phys 3739

Hamaya N and Akimoto $\mathbf{S} 1981$ High Temp. High Pressures 13347

Hanneman R E 1969 in Reactivity of solids (ed.) J W Mitchell et al (New York: Wiley Inlerscience) pp 789 Kutsar A R, German V N and Nosoya G I 1974 Sou. Phyr. Dokl 18133

Laczm A. Peyronneau J and Leliwa Kopystynski J 1973 J. Physique 341055

Landauer R 1952 J. Appl. Phyr. 23719

Leliwa-Kopystynski J, Peyronneau J and Lacam A $1975 \mathrm{~J}$. Physique 361273

Livstitz L D, Genshaft Yu Sand Ryabinin Yu N 1960 Fiz. Met. Metalloved 9126

Livstitz L D, Ryabinin Yu N. Larionov L V and Zverev A S 1969 Sov, Phyr. JETP 26612

Lombos B A and Mahdaly H M 1917IEEETrans. Geosci. Electron GE-15 203

MeQueen R G and Marsh S P 1960 J. Appl. Phys. 311253

McQueen R G, Marsh S P, Taylor I W, Fritz I N and Carter W J 1970 High-velocity impact phenomena (ed.) R

Kinslow (New York Academic Press) p. 341

Murali Mohan, Divakar $C$ and Singh A K 1982Trans. Indian Inst. Met, 35 (Special No) p. I

Onodera A 1971 Rev. Phyr Chem. Jpn 41 I

Osugi J, Harz K and Katayama M 1975 Bull. Inst. Chem Res. Kyoto Univ $\mathbf{5 3} 269$

Ray $\boldsymbol{R} 1969$ in Reactivity of solids (ed.) J W Mitchell et a (New York Wiley Interscience) pp 777

Samara G A, Walters L $C$ and Northrop D A 1967 J. Phys. Chem. Solids 281857

Singh A K, Murali Mohan and Divakar C 1982a J. Appl. Phys, 521221

Singh A K. Divakar C and Murali Mohan 1982b Tweaty thind HighPtessure Conference of Japan, Kyoto,

Extended Abstracts, pp 100

Singh A K, Divakar C and Mohan M 19839 Rev. Sci. Instrum. 54 (to appear)

Singh A K, Murali Mohan and Divakar C 1983b J. Appl. Phyr 54 (to appear)

Takano K, Wakatsukj M and Tabata K 1982 Twenty third Higb Pressure Conferede of Japan, Kyoto.

Extendod abstracts pp 14

Vobra Y K, Sik \$a S, Vaidya S N and Chidambaram R 1977 J. Phyr Chem Solids. 381293

Zeto R J and Roy R 1969 in Reactivity of solids (ed.) J W Mitchell et al (New York: Wiley Interscience) pp 803

Zeto R J. Vantlet HB, Hryckowian Eand Bosco C D 1968 Accurate characterization of the high-pressure

environment NBS Special Pubi No. 326 (ed.) E C Lloyd (Washington DC: Govt Printing Ofioe) pp 25

Zeto $R \mathrm{~J}$ and Vanflet H B 1969 J. Appl. Phyr. 402227

Zil'Bershteyn VA, Chis totina N P, Zharov A A, Grishina N Sand Estrin EI 1916Phys. Metallogr.(USSR) 39 208

Zil'Rershteyn V A, Nosova G I and Estrin E I (1974) Phys Met. Metallogr. (USSR) 35128 